(Aus dem physiol. Institute der Universität Wien.)

\title{
Die Veränderungen der Genitalschleimhaut während der Gravidität und Brunst bei einigen Nagern.
}

Von

Dr. Hans Königstein, Wien.

In einer Arbeit über das Schicksal der nicht ejakulierten Spermatozoen ${ }^{1}$ ) gelang es mir, eine Reihe von Tatsachen anzuführen, welche geeignet sind, die Vermutung S. Exner's ${ }^{2}$ ) über die Resorption der Samenfäden in der menschlichen Samenblase zu stützen. Es lag nun nahe, auch die Veränderungen zu verfolgen, denen die Spermatozoen im weiblichen Genitaltrakt unterworfen sind. Da die weiblichen Nager, an denen diese Versuche unternommen wurden, ausschliesslich zur Zeit der Brunst, die kaum 24 Stunden dauert, den Geschlechtsverkehr gestatten, so ist das Schicksal der Spermatozoen an diesen Termin gebunden. Es ergaben sich nun mannigfache Wechselbeziehungen zwischen den Verwandlungsformen der Samenfäden und den Schleimhautveränderungen des Genitaltraktes zur Zeit der Brunst; daher scheint es angezeigt, die Beschreibung einiger Erscheinungen, welche die Brunst im weiblichen Genitale hervorruft, zusammenfassend zu beschreiben und vorauszuschicken.

Die in den Laboratorien zur Beobachtung kommenden weiblichen Nager lassen unmittelbar nach dem Wurf die Begattung zu, sind also brünstig. Erfolgt keine Konzeption, so tritt in drei- resp. vierwöchentlichen Intervallen wieder Brunst ein. Die Brunst erseheint demnach in zwei Formen, entweder unmittelbar in der Gefolgschaft der Gravidität, so dass die Vorstadien der Brunst mit den Phasen

1) Archiv f. d. ges. Physiol. Bd. 114 S. 199.

2) Die Physiologie der männlichen Geschlechtsfunktionen. Handbuch der Urologie, herausg. von Anton v. Frisch und Otto Zuckermandl, Bd. 1 S. 234-236. 
der vorgeschrittenen Trächtigkeit zusammenfallen müssen, oder vollkommen unabhängig von der Fruchtentwicklung.

Die vorliegende Untersuchung schildert einige Veränderungen des weiblichen Genitalschlauches und versucht, aus der Reihe der verschiedenen Wandlungsformen, die den wechselnden Funktionsstadien der Gravidität entsprechen, speziell jene Bilder festzuhalten, welche im ursächlichen Zusammenhange mit der Brunst stehen. Zugleich sollen die unter dem genannten Gesichtspunkte erhobenen Befunde aus dem Gesamtkomplex der Graviditätsveränderungen herausgehoben und den reinen Brunstformen gegenübergestellt werden.

Es hat sich im Laufe der Untersuchung als notwendig herausgestellt, die Beobachtungen nicht auf den Uterus, wie dies meist geschehen ist, zu beschränken, sondern auch Vagina, Cervix und Tuben sowie die Schleimhautprodukte gleichmässig in den Kreis der Betrachtungen zu ziehen, da im ganzen Genitaltrakt Erscheinungen auftreten, die im Prinzip gleichartig zu bewerten sind. Zum leichteren Verständnisse möchte ich hier die Resultate dieser Arbeit, die später im Detail ausgeführt werden, kurz hervorheben.

An der Schleimhaut der Vagina und der Cervix spielt sich in bestimmten Perioden ein Zyklus von Form- und Funktionsveränderungen des Epithels ab, der zu einer teilweisen Verschleimung derselben führt.

Bei der Vagina kommt nur die dem Uterus zugekehrte obere Hälfte in Betracht, und es ist dies hervorzuheben, da sich ihre beiden Abschnitte sowohl makroskopiseh als mikroskopisch deutlich voneinander unterscheiden.

Bei dem geschilderten Prozesse wechseln Platten- und Zylinderepithelien miteinander ab. Der übereinstimmende Typus des Vorganges, der sich an der Schleimhaut der Vagina und der Cervix vollzieht, bleibt gewahrt, obwohl das Epithel während der Genitalruhe in der Scheide ein Plattenepithel, an der Cervix ein zweischichtiges Zylinderepithel ist.

Die periodischen Veränderungen der Scheiden und Cervixschleimhaut werden zwar von der Gravidität modifiziert, treten jedoch in vielen Punkten übereinstimmend auch unabhängig von derselben auf und scheinen in ursächlichem Zusammenhang mit der Brunst zu stehen.

Die erwähnten Epithelverwandlungen werden von einer Leukozytose begleitet, die sich über den ganzen Genitaltrakt bis zur Einmündung der Tuben in die Uterushörner erstreckt und nicht nur 
für den Umbau der Schleimbaut in Betracht kommt, sondern auch in ihrer Beziehung zu den im Genitaltrakt befindlichen Spermatozoen sowie zu dem Ei von dem Augenblick seines Eintrittes in das Uterushorn bis zum Momente der Implantation berücksichtigt werden muss. Es ist selbstverständlich, dass bei der Verschleimung der Zellen der produzierte Schleim sich dem Inhalte des Genitalkanals beimengt und die besondere Reichlichkeit desselben zu bestimmten Zeiten bedingt. Abgesehen vom Schleime konnte jedoch noch die Produktion eines eosinophylen Sekrets an den Cervixzellen nachgewiesen werden, so dass wir zwei Produkte derselben zur Zeit der Brunst zu unterscheiden haben.

Bezüglich des Untersuchungsmaterials erwähne ich, dass mir neben weissen Ratten in Genitalruhe eine geschlossene Reihe der verschiedensten Graviditätsstadien sowie des Puerperiums, im ganzen 18 Exemplare, und endlich eine Brunstratte zur Verfügung standen. Dabei ist $\mathrm{zu}$ bemerken, dass unter Genitalruhe jener rasch vorübergehende Zustand $\mathrm{zu}$ verstehen ist, in welchem die Uterushörner auf ihr Minimum zurückgebildet sind, also keine Graviditätserscheinungen, andererseits aber auch keine Anzeichen der Brunst bestehen. Ausserdem konnte ich eine trächtige und eine läufige Hündin und ein Meerschweinchen sowie fünf Kaninchen in verschiedenen Stadien untersuchen. Der in Zenker'scher Flüssigkeit fixierte Genitaltrakt wurde mit Rücksicht auf die vorzunehmenden Zellstudien in dünne, $4-5 \mathrm{~m}$ betragende Sehnitte zerlegt, wobei von den Tuben vollständige, vom übrigen Genitale Stufenserien hergestellt wurden. Es bleibt eine Lücke dieser Untersuchung, deren Ausfüllung jedoch noch längere Zeit in Anspruch nehmen würde, dass die einzelnen Stadien der einfachen Brunst nicht näher untersucht werden konnten, und ich mich daher mit einem allgemeinen Vergleich der beiden Brunstformen begnügen muss.

Die Arbeiten über den graviden Uterus und über die Menstruation sind zu einer grossen Literatur angewachsen, die nur sehr schwer zu überblicken ist. Ich glaube jedoch mit dem Hinweis auf den bereits erwähnten speziellen Grundgedanken dieser Untersuchung nur jene Arbeiten erwähnen zu müssen, die in einem direkten Zusammenhang mit den von mir erhobenen Befunden stehen.

Bei der Beschreibung meiner Präparate werde ich mit der Vagina beginnen, die Schleimhautveränderungen gegen die Tuben hin verfolgen und erst am Schlusse auf die Beschaffenheit des 
Genitalinhaltes zu sprechen kommen. Dabei werde ich den histologischen Befunden eine kurze makroskopische Beschreibung vorausschicken, teils um die untersuchten Stadien zu charakterisieren, teils um einige auffällige Befunde hier zu erwähnen.

Da ich bei der weissen Ratte ebensowenig wie manche andere Autoren den Koitus beobachten konnte, habe ich bei der Berechnung der Tragzeit den Termin des Wurfes, dem die Kohabitation innerhalb der nächsten 24 Stunden gefolgt sein musste, herangezogen oder die Grösse der Embryonen zur Bestimmung benützt.

\section{Vagilla.}

Bei der Ratte ist wäbrend des Ruhezustandes des Genitales der Eingang in die Vagina ebenso wie während des grössten Teils der Gravidität geschlossen. Nur wenige Tage vor dem Wurfe sah ich manchmal den Scheideneingang klaffen, ebenso bei einer vor längerer Zeit ovariotomierten Ratte.

Die Vagina des unbefruchteten Tieres weist zarte Längs- und nur mit der Lupe wahrnehmbare Querfältchen auf, ohne dass hier ein wesentlicher Unterschied zwischen dem uterinen und dem vulvären Abschnitt getroffen werden könnte. Während der Gravidität nehmen die Maasse für die Länge wie für die Weite der Vagina ziemlich rasch zu, so dass schon im ersten Drittel derselben mit Sicherheit eine Wachstumszunahme festgestellt werden kann. Allmählich werden die Falten im vulvären Abschnitt breiter und höher, und das Aussehen des uterinen Anteiles ist insofern verändert, als die Schleimhaut in ihrer Gesamtheit dicker wird. Die Furchen zwischen den Falten sind durch Schleim ausgefüllt und erst nach Entfernung desselben lässt sich auch hier eine Volumzunahme der Längsfalten und ein deutlicheres Hervortreten der Querfalten wahrnehmen. Mit der Schilderung dieses Stadiums, welches etwa der Mitte der Gravidität entspricht, sind bereits die makroskopisch auffindbaren Unterscheidungsmerkmale der beiden Teile der Vagina hervorgehoben, nämlich eine unverhältnismässige Dickenzunahme der oberen Schleimhautanteile sowie eine von dort ausgehende Schleimproduktion. Nach der Geburt ist die ganze Scheide mit Schleim ausgegossen.

Die Scheide des Meerschweinchens spiegelt dieselben Verhältnisse, nur entsprechend ihren grösseren Dimensionen in charakte- 
Die Veränderungen der Genitalschleimhaut während der Gravidität etc. 557

ristischerer Weise wieder, während beim Kaninchen ebenso wenig eine erhebliche und bestimmt lokalisierte Schleimproduktion wie irgendein anderes Merkmal auftritt, welches die Beschreihung zweier getrennter Abschnitte rechtfertigen würde. Die Falten der Vagina des Kaninchens sind im allgemeinen niedrig, namentlich niedriger als die Erhebungen der Cervix, die noch später näher gewürdigt werden sollen.

Die Vagina des Kaninchens weicht von den eben beschriebenen noch durch eine Besonderheit ab. Sie besitzt nämlich eine etwa $2 \mathrm{~mm}$ hohe Falte, die zwischen beiden Portiozapfen von der ventralen gegen die dorsale Seite zieht. Wenn dieselbe auch nicht hoch genug ist, um die eine Portio vollständig von der anderen zu scheiden, so grenzt sie jedoch in der Region der Scheidengewölbe für jeden Uterus ein eigenes Receptaculum seminis ab.

Die nun folgenden Schilderungen über die periodisch ablaufenden Veränderungen in der Vagina der Nager können sich teilweise auf die Angaben früherer Autoren stützen. Denn die Tatsache der Verschleimung des Epithels ist in den Arbeiten von Moreau ${ }^{1}$ ), Salviole ${ }^{2}$, Lataste $^{3}$ ) und Retterer ${ }^{4}$ ) bereits unzweifelhaft festgestellt worden, doch weichen die Ansichten der beiden letztgenannten Autoren insofern auseinander, als Lataste die Gravidität als Ursache für die Verschleimung ansieht, während R et te r er den periodischen Ablauf des Prozesses von der Ovulation abhängig macht und der Gravidität bloss einen modifizierenden Einfluss auf die Abwickelung der Veränderungen zuerkennt.

Die Berechtigung für die nochmalige Beschreibung dieser Veränderungen scheint mir einerseits in dem Gegensatz, der zwischen den Meinungen früherer Autoren besteht, zu liegen, andererseits können in dieser Arbeit auch einzelne Details dem Bekannten hinzugefügt werden. Schliesslich ist die Besprechung der Vorgänge in der Vagina zum Verständnisse für den Umbau in der Cervix, der, soviel ich glaube, noch nicht gewürdigt wurde, notwendig.

1) Des transformations épithéliales de la muqueuse du vagin de quelques rougeurs. Journal de l'Anatomie 1889.

2) De la structure de l'épithélium vaginal de la lapine et des modifications qu'il subit pendant la gestation. Arch. ital. de Biologie t. 17. 1892.

3) Rhythme vaginal des mammifères. Compt. rend. de la societé de biol. 1893.

4) Sur la morphologie et l'évolution de épithélium au vagin des mammifères. Compt. rend, de la société de biologie 1892. 
Zur genauen Klarlegung der Umwandlungen, denen das Vaginalepithel unterworfen ist, müssen wir an dem Gedanken festhalten, dass das mehrschichtige Plattenepithel die Grundform darstellt, von der alle Veränderungen ihren Ausgang nehmen, um wieder zu ihr zurückzukehren. Dieser Gedanke findet seine Stütze in dem Umstande, dass auch bei Genitalruhe Plattenepithel der vaginalen Schleimhaut ihren Charakter verleiht. Andererseits muss jedoch hervorgehoben werden, dass dieser Ruhezustand nur ein vorübergehender ist, und dass das Ausbleiben der Gravidität keineswegs ein Bestehenbleiben des Plattenepithels bedingt. Vielmehr geht auch in diesem Fall ein Umwandlungszyklus vor sich.

Bei Ratten, bei welchen eine Gravidität von der nächsten unmittelbar abgelöst wird, ist das Plattenepithel eine ausserordentlich flüchtige Erscheinung. An Tieren, welche 24-36 Stunden nach dem Wurfe getötet wurden, sieht man fast in der ganzen Ausdehnung der Vagina mehrschichtiges Plattenepithel; doch geben noch Reste von Zellen, die zum grössten Teil abgelöst sind, zum Teil aber noch festhaften und die alle deutlich verschleimt sind, Zeugnis für die Verschleimung $a b$, die noch vor 24 Stunden vorgeherrscht hat. Andererseits haben bereits meist mehrkernige Leukocyten aller Orten das Plattenepithel durchsetzt und sind bis an die obersten Schichten vorgedrungen. In diesen baben wir die Vorboten der baldigen Zerstörung dieses Epithels zu erblicken.

Tatsächlich ist auch die Abstossung des Plattenepithels 4 Tage nach dem Wurfe bereits vollständig vollzogen und die Bildung eines abermals neuen Epithels im Gang. Immerhin kann man an diesen Stadien noch die Formen studieren, unter denen der Epithelwechsel sich vollzieht. Durch die Ablösung der Zellen in der obersten Schichte kommt es zur Bildung von Cysten, die einerseits durch hineingelangte Zellen erfüllt werden, andererseits durch neue Ablösungen an Grösse zunehmen.

Die basalen Zellen bilden stets die Grenze, an der die Ablösung der Zellen Halt macht und sind zugleich der Mutterboden für ein mehrreihiges Epithel, dessen oberste Schichte aus Zellen besteht, die allmählich hoch und schmal werden und dadurch den Charakter des Zylinderepithels gewinnen.

Sehr bald, schon am Ende der ersten Woche, also wenige Tage nach der Entstehung dieser neuen Zellgeneration setzen die Vorgänge der Verschleimung, die zunächst wesentlich die mittleren Zellschichten 
Die Veränderungen der Genitalschleimhaut während der Gravidität etc. 559

betreffen, ein. Dabei nehmen die Zellen dieser Lagen immer mehr an Volumen zu, bis sie jene unförmige Gestalt erreicht haben, welche dem Vaginalepithel in den letzten zwei Dritteln der Gravidität das charakteristische Gepräge verleihen. Während dieses Verschleimungsprozesses werden die basalen Zellen auf einen schmalen Streifen zusammen gedrängt, ihre Kerne treten näher aneinander heran, und die Zellen, welche an die verschleimten Partien grenzen, sind deutlich platt gedrückt. Die oberste Zellreihe, welche dem Lumen zugekehrt ist, scheint lange an der Verschleimung keinen wesentlichen Anteil zu nehmen, und bewahrt bis kurze Zeit vor dem Wurf unverändert die Zylinderzellengestalt. Zur Zeit des Wurfes verschleimen auch diese Zellen. Zugleich mit dem Ende der Gravidität erreicht auch die Verschleimung ihren Höhepunkt. Um dieses Stadium zu kennzeichnen, muss der gegebenen Schilderung bloss noch hinzugefügt werden, dass die verschleimten und aufgetriebenen Zellen so an Umfang zunehmen, dass ihr Kern häufig nicht mehr in die Schnittebene fällt, wodurch die Bildung grosser Inseln scheinbar kernloser Zellen erklärt wird.

Die Verschleimung ist gerade in den Buchten und Furchen der Schleimhaut eine besonders reichliche und ausgedehnte und führt zur fast vollständigen Ausfüllung derselben. Die Erhebung dieses Befundes bildet eine Ergänzung der bereits makroskopisch gemachten Beobachtung, der zufolge die Unebenheiten des oberen Vaginalanteiles bei vorgeschrittener Schwangerschaft durch Schleimmassen ausgeglichen werden.

Obwohl schon in der zweiten Hälfte der Schwangerschaft eine Desquamation und Ablösung des Epithels stattfindet, kommen für die Abhebung in grossen Massen doch erst die Gewaltseinwirkungen der Geburt und des Koitus als mechanische Momente in Betracht. Die Betonung dieser eben genannten Momente gibt auch für das Zustandekommen des noch später zu besprechenden Vaginalpfropfens eine befriedigende Erklärung.

Alle früher genannten Autoren heben hervor, dass zur Zeit der einfachen Brunst die Vagina bei Maus und Ratte mit mebrschichtigem Plattenepithel bekleidet ist. Dieses Epithel wird, wie Lataste ${ }^{1}$ ) bemerkt, sofort abgestossen, und ein zylindrisches verschleimendes tritt an seine Stelle.

1) 1. c. Nr. 3 S. 557. 
Bei meiner Ratte, die kurz nach der Brunst, ohne belegt worden zu sein, getötet wurde, war das Plattenepithel abgefallen, und sie bot folgendes Verhalten.

In der Vagina findet sich ein niedriges, stellenweise bloss einreihiges Zylinderepithel mit den Zeichen der Verschleimung und der Desquamation. Wir sehen demnach, dass auch bei dieser Ratte, die vom Männchen isoliert gehalten wurde und bei der eine Konzeption auszuschliessen ist, eine Umwanderung des Plattenepithels in verschleimendes Zylinderepithel erfolgt ist. Die Verschleimung und Desquamation verraten uns die Tatsache, dass in diesen Präparaten bloss ein vorübergehender Funktionszustand festgehalten wurde, der bei normalem Verlauf der Genitalruhe und damit der Ftablierung von Plattenepithel Platz gemacht hätte. Wir können demnach behaupten, dass der periodische Wechsel der Bilder, welche die Vaginalschleimhaut bietet, unabhängig von der Gravidität seinen bestimmten gesetzmässigen Verlauf nimmt.

In diesem Zyklus folgen Plattenepithel, Zylinderepithel und Versehleimung aufeinander. Der Unterschied, der durch die Gravidität hervorgerufen wird, gibt sich nur in einer Verlängerung der Verschleimungsperioden zu erkennen. Durch diese wird bei vorausgegangener Gravidität die Plattenepithelbildung hinausgeschoben. Während bei der einfachen Brunst Plattenepithel für die Vagina charakteristisch ist, folgt diese Bildung bei vorausgegangener Gravidität um einen Tag verzögert nach.

Bei einem Meerschweinchen in der achten Woche der Gravidität und bei einem, das 24 Stunden nach dem Wurfe untersucht wurde, fand sich übereinstimmend eine bochgradige Verschleimung des Vaginalepithels, ohne dass das Präparat aus der Zeit nach dem Wurfe, wie das bei der Ratte der Fall ist, die Ansätze einer Plattenepithelentwicklung erkennen liesse. Die Beobachtung über das $\Lambda$ usbleiben dieser Zeliform deckt sich mit den Anschauungen Ret ter e $\mathrm{r}^{\mathrm{s}} \mathrm{s}^{1}$ ), der das Persistieren eines verschleimten Zylinderepithels während des Rubezustandes und während der Gravidität beschreibt.

Meine Beobachtungen an Kaninchen kommen für die Frage der Brunstveränderungen nicht weiter in Betracht, mögen jedoch zum Vergleich mit der Ratte und zur Feststellung einzelner histologiseher Details hier erwähnt werden.

1) l. c. Nr. 4 S. 557. 
Die niedrigen und zarten Falten der Vagina zeigen seitliche Leistchen und tragen ein durchweg einreihiges Zylinderepithel. Bei den untersuchten Tieren, bei welchen der letzte Koitus 2, 3,6 und 8 Tage zurück datierte, zeigten die Zylinderzellen verschiedene Stadien der Sekretion, niemals jedoch irgendein Zeichen von Zelldesquamation.

Die Gestalt und die Färbbarkeit der Zellen wechselt mit den Stadien der Sekretion. Bei dem Kaninehen, das 6 Tage nach dem Wurf und dem anschliessend daran erfolgten Koitus getötet wurde, erreichen die Zellen mehr als die doppelte normale Höhe und sind auch in der Breitendimension vergrössert. Während das Protoplasma in den normalen Fällen gleichmässig und intensiv mit Eosin färbbar ist, weisen die Leiber dieser Zellen im H. E.-Präparat eine sehr wenig intensive, leicht grau rötliche Färbung auf, wobei eine deutliche wabige Struktur des Protoplasmas zutage tritt. Der Kern ist vollständig an die Basis der Zelle zurückgetreten. Die Zellen geben keinerlei Schleimfärbung. Dieselbe wurde übrigens auch in dem geringen flüssigen Inhalte der Scheidengewölbe vergebens gesucht. Die Zellen zeigen demnach zwar Erscheinungen der Sekretion, produzieren jedoch keinen Schleim und zerfallen nicht. Diese Tatsache muss gegenüber der Ratte mit besonderer Betonung hervorgehoben werden. Zugleich sei hier an die Sektionsbefunde erinnert, die bei der Ratte dicken Schleim, beim Kaninchen nur eine geringe Menge flüssigen Inhalts in der Vagina ergaben. Indem ich hier und anderwärts das Verhalten des Zellinhalts oder der Vaginaflüssigkeit gegenüber dem Hämatoxylin, dem Thionin und dem Muchämatein als Kriterium für die Schleimnatur derselben auffasse, folge ich dem Gebrauch der Histologen, und muss die Verantwortung für die Stichhaltigkeit dieses Kriteriums auch diesen überlassen.

\section{Cervix.}

Ratte. Die Cervix unterliegt während der Gravidität zunächst den typischen Veränderungen. Die Portio wird plumper, der Cervicalkanal kürzer - seine Länge sinkt bis zu $3 \mathrm{~mm}$ herab - und bedeutend weiter, so dass er am Ende der Gravidität einen dreimal so grossen Breitendurehmesser ausweist; als zum Beginn derselben. $\mathrm{Zu}$ gleisher Zeit damit entwickeln sich die Schleimhautfalten in grosser Anzahl und zu bedeutender Höhe.

Von Interesse sind jene bei den Ratten sehr häufigen Fälle, in welchen eine tiefe Inplantation des untersten Eichens stattgefunden 
hat, da hierbei ein Teil der Cervix zur Aufnahme der Frucht herangezogen wird. Bei diesen tiefsitzenden Eiern fällt auch der Scheidewand zwischen den beiden Gebärmutteranteilen eine wesentliche Rolle zu. Diese Wand, deren unterer Anteil noch der Cervixregion. angehört, während der obere Anteil aus dem Gewebe zwischen den Uterushörnern hervorgeht, wird unter den vorausgesetzten Umständen in eine papierdünne, durchsichtige Membran umgewandelt. Dieselbe besitzt ein äusserst zartes, bindegewebiges Stroma, das neben vereinzelten Gefässen wenige Muskelbündel führt. Diese Muskelzüge sind der letzte Rest eines mächtigen Muskelpaketes, das unter normalen Verhältnissen in diese Wand eingelagert ist und dadurch zustande kommt, dass hier die zwei Längs- und Ringmuskelschichten beider Uterushälften hart aneinandergrenzen. Die Oberflächen der Membran sind den beiden gegenüberliegenden Fruchtkammern zugekehrt und mit einschichtigen Plattenepithelzellen bedeckt.

Während bei der Ratte die Uterushälse beider Seiten eng verbunden sind und in Form eines Zapfens, der beide Kanäle führt, in die Vagina hineinreichen, beginnt bei den $\mathrm{Kaninchen} \mathrm{der} \mathrm{Zu}-$ sammenhang der beiden Uterushälften erst jenseits der Grenzlinie, die durch das Herantreten der vaginalen Wand an den Uterushals. gebildet wird. Die beiden Portioanteile ragen demnach getrennt in das Fornixbereich hinein und dabei erhebt sich in dem Zwischenraum, den sie in der Mitte freilassen, die bereits beschriebene Falte.

Durch zahlreiche, tiefere und oberflächlichere Furchen, welche radiär verlaufen, und bis gegen das Lumen ziehen, um im Cervicalkanal ihre Fortsetzung zu nehmen, erscheinen die Portiozapfen gelappt. Die eben beschriebene, auf der Aussenseite sichtbare Faltenbildung tritt im aufgeschnittenen Kanal nur noch mit grösserer Deutlichkeit hervor, nimmt jedoch gegen das Uterushorn hin an Mächtigkeit wieder ab. Die Falten des Cervixkanals sind sowohl längs wie quer verlaufend.

Die histologischen Erörterungen, die nun folgen, werden mehrfach an die bereits gegebene Beschreibung der Vagina erinnern, sowohl durch die Gesetzmässigkeit, die dem Wechsel der Erseheinungen anhaftet, als auch durch bestimmte Charaktere des Umbaues, wie die Verschleimung und die Bildung von Plattenepithelzellen. Im allgemeinen ist die Gesamtheit der Veränderungen eine weniger hochgradige, der Ablauf der einzelnen Phasen vollzieht sich rascher und die Zeit der Epithelruhe ist daher eine längere. 
Als neue Erscheinung tritt uns in der Cervix der Ratte die Bildung zweier Zellprodukte von versehiedener Struktur und verschiedener Färbbarkeit entgegen.

Der Verlauf der Zellveränderungen ist folgender: am Ende der ersten Woche nach dem Wurf ist die Cervix in ihrer ganzen Ausdehnung mit einem zweireihigen Zylinderepithel, welches auch dem Normalzustand bei Genitalruhe entspricht, ausgekleidet. Dabei findet man in der Tiefe der Falten und zwar besonders zahlreich in den oberen Abschnitten kleine und grosse eosinophile Sekrettropfen. Dieses Sekret bildet die eine Art der Zellprodukte, während bei der gleich zu erwähnenden Verschleimung die zweite Art derselben entsteht.

Das geschilderte Verhalten ändert sich bei eingetretener Gravidität erst in der Mitte der Tragzeit, insofern einzelne Zellen verschleimen und zerfallen und dureh diesen Prozess grosse und kleine zystische Lücken zwischen den Epithelzellen bilden, die mit Zellkernen, Schleim und Leukocyten erfüllt sind. Nur allmählich schreitet dieser Prozess, gekennzeichnet durch immer zahlreicher und grösser werdende schleimgefüllte Cysten, fort.

Kurz vor dem Wurf ist ein plötzlicher Anstieg der Verschleimung zu bemerken, und gleich nach dem Wurf liegt ein mehrschichtiges, grösstenteils verschleimtes Zylinderepithel vor. Die Zellen, die durch Schleimmassen unförmig aufgetrieben wurden, sind sehr zahlreich, und doch deuten bereits viele Zellen, die in wohlerhaltener Gestalt abgestossen werden, so wie Zelltrümmer und polynucleäre Leukocyten auf eine beginnende Neugestaltung hin.

Diese Präparate sind noch in einer weiteren Hinsicht, nämlich für das Studium der Zellfunktionen in der Cervix wäbrend der Brunst von Interesse. Aus der Anzahl jener Zellen, welche für diese Frage in Betracht kommen, fallen uns in erster Linie einige auf, die in der obersten Reihe ihren Platz haben und durch ihre besondere Gestalt von der Norm abweichen.

Der Kern, der keine Besonderheiten erkennen lässt, liegt im oberen Zelldrittel und bildet zugleich die Grenze für zwei vollkommen verschiedene Zellanteile. Die randständig vor dem Kern gelegene Partie ist zylindrisch gebaut und ziemlich gleichmässig intensiv mit Eosin gefärbt. Und nur bei starker Vergrösserung erscheint das Protoplasma nicht vollkommen homogen, sondern andeutungsweise gekörnt. Der basalwärts gelegene Abschnitt ist keulen- 
förmig aufgetrieben, und wie ich auf Grund verschiedener Färbungen schliessen kann, mit Schleim gefullt, in dem hier und da einzelne Vakuolen zu sehen sind. Es darf nicht unerwähnt bleiben, dass man auch an Schiefschnitte denken muss, durch die der Zusammenhang zwischen dem schleimgefüllten basalen und dem eosinophylen randständigen Zellanteil vorgetäuscht wird, während tatsächlich der basale Teil einer tieferen Zellschicht angehört. Ich habe jedoch die beschriebene Zellform nicht nur wiederholt beobachtet, sondern gerade auch an Präparaten gesehen, bei welchen an der ganzen Zellbegrenzung des Lumens die Zellen gleichmässig getroffen waren, also Schiefschnitte unwahrseheinlich sind. An anderen Zellen ist die Gestalt nicht wesentlich verändert, hingegen zeigt das mit Eosin gutgefärbte Protoplasma zum Unterschied zu den eben genannten Formen einen lockeren, wabigen Bau und führt als Übergangsstufe zu einem dritten Funktionszustand der Zellen, der durch eine ziemlich gleichmässige Volumzunahme und durch ein eigenartiges Protoplasma ausgezeichnet ist. Dasselbe ist grösstenteils ungefärbt, erscheint durchsichtig und enthält in ziemlich grossen Abständen zahlreiche rotgefärbte, feinste Körnchen, die ihm ein punktiertes Aussehen verleihen.

Diese geschilderten Zellen wurden als markanteste Typen herausgehoben, zwischen die sich eine ganze Reihe Zellen mit mehr oder weniger aufgelockertem Protoplasma als Übergangsformen einschalten lassen, die uns den Weg von dem fast homogen gefärbten bis zum ungefärbten Protoplasma, das gefärbte Körnchen enthält, führen.

Die vorliegende Beschreibung ist nach H. E.-Präparaten gegeben. Einzelne Schnitte wurden auch nach Heidenhain gefärbt, ohne jedoch geänderte Details zu bieten.

Auf Grund dieser zu wiederholten Malen gemachten Beobachtung darf wohl der Vermutung Ausdruck gegeben werden, dass die Cervixzellen nach dem Wurfe ausser dem bereits sichergestellten Schleim noch ein anderes körniges eosinophyles Produkt sezernieren, und dass ich diese sezernierenden Zellen in verschiedenen Phasen ibrer Funktion vor Augen gehabt habe. Die Beobachtung einer feingekörnten eosinophylen Masse, die neben typischem Schleim sich im Inhalte des Kanales vorfindet, kann als Stütze für diese Ansicht angeführt werden. Andererseits bleibt noch eine zweite, jedoch weniger wahrscheinliche Deutung dieser Befunde zu erwähnen. Der Zweck der Protoplasmaauflockerung könnte auch darin gelegen sein, dem Schleim, der in 
der Zelle produziert wird, einen Austritt zu ermöglichen; da jedoch die versehleimten Zellen auch zerfallen, fehlt zumindestens die No:wendigkeit für diesen Weg der Entleerung, ausserdem bliebe in diesem Falle auch die Bedeutung der roten Körnchen unaufgeklärt.

Diese Zellen haben hier wegen ihres histologiscben Interesses Erwähnung gefunden und an ihre Beschreibung wurde eine Vermutung über ihren Funktionszustand geknüpft. Sollte sich diese Ansicht nicht bewahrheiten, so besteht immer noch die Annahme zu Recht, dass in der Cervix neben dem Schleim noch ein eosinophyles Sekret geliefert wird, da, wie ich eingangs bemerkte, in den Buchten der normalen Schleimhaut zahlreiche derartige Sekrettropfen abgelagert werden.

An den Präparaten, welche die Verhältnisse etwa 4 Tage nach dem Wurfe darstellen, ist die Bildung von neuem Schleim fast vollständig eingestellt, und einzelne noch wahrnehmbare Reste desselben stammen aus dem früheren Stadium. Hingegen treten alle Anzeichen, welche für den Abbau des alten und für die Entstehung eines neuen Epithels zeugen, in den Vordergrund. Stellenweise ist das Epithel zylindrisch und ist in zwei Reihen angeordnet. An anderen Orten kam es zur Bildung von Plattenepithelien, überall aber ist das Epithel von zahlreichen kleinen und grossen Cysten, mit verschiedenem Inhalte, durchsetzt.

Die Betrachtung der Cysten gewährt uns einen Einblick in die einzelnen Vorgänge, die der Wiederherstellung des zweireihigen Zylinderepithels, welches für den Ruhezustand charakteristisch ist, vorausgehen. An zahlreichen Stellen entstehen die Cysten durch Abhebung der oberflächlichen Zellschichten und durch Nachrücken tieferer Zellen in den entstandenen Hohlraum. In diesen Fällen besteht der Cysteninhalt neben Leukocyten, den steten Begleitern dieses Prozesses, aus wohlerhaltenen Epithelzellen. Anderenorts sieht man am Rande dieser Cysten gequollene, schlecht färbbare, im Zerfall begriffene Zellen, im Lumen derselben Detritus, eventuell rote Sekrettropfen. Hier scheint der Zellverfall die Entstehungsursache der Cysten zu sein.

Fügen wir dieser Beschreibung noch hinzu, dass unter den abgestossenen Zellen die neugebildeten Plattenepithelien sehr zahlreich vertreten sind, so gewinnen wir die Überzeugung, dass dieser Zelltypus bei dem vorsichgehenden Umbau nur als kurzdauernde Übergangsform in Betraclit kommt. Immerbin aber vollzieht sich nach 
der Verschleimung, die der Zeit vor und kurz nach dem Wurfe angehört, die Wiederherstellung des zweireihigen Zylinderepithels, auf dem Wege der Plattenepithelbildung.

Die Untersuchung der Brunstratte ist nun insofern interessant, als sich auch dort in der Cervix vereinzelte schleimgefüllte Cysten finden, die den Vergleich mit einem Stadium kurz vor dem Wurf gestatten.

Nach Erwägung aller bisher gemachten Beobachtungen kommen wir zu folgenden Resultaten: Sowohl in der Vagina wie in der Cervix laufen zyklische Epithelveränderungen $a b$, und gerade der Umstand, dass diese Veränderungen auch während der reinen Brunst auftreten, weist auf ihre Unabhängigkeit von der Gravidität hin und macht ihren Zusammenhang mit der Brunst wahrscheinlich.

In der Cervix des Kaninchens fällt die besonders reichliche Faltenbildung auf. Es finden sich dort nicht nur hohe Falten, die seitliche Leisten tragen, sondern die letzteren streben wieder ihrerseits durch Leisten dritter Ordnung eine weitere Oberflächenvergrösserung an. Gegen das Uterushorn hin werden die Falten breiter, verzichten aber auf seitliche Sprossenbildungen. Das Epithel ist ein einreihiges Zylinderepithel mit Flimmerhaaren, die einem Basalkörperchen aufsitzen. Letztere Tatsache konnte an HeidenhainPräparaten festgestellt werden. Am 8. Tag nach erfolgter Konzeption sind die Zellen stark aufgetrieben und zeigen ein vollkommen durchsichtiges Protoplasma, das keinerlei Färbung annimmt, auch die Schleimreaktion nicht gibt. Die Volumvergrösserung wird wohl auf eine Sekretanhäufung zurückzuführen sein. Wesentlich ist noch die Bemerkung, dass weder nach dem Wurfe noch nach erfolgter Konzeption in der Cervix so wenig wie in der Vagina Zeichen einer Desquamation zu bemerken sind, worin ein deutlicher Unterschied gegenüber der Ratte liegt.

An Serienschnitten, die durch den Meersehweinchen-Uterus nach dem Wurfe gelegt sind, erkennt man, dass das verschleimende Epithel der Vagina nach wenigen Schnitten, an denen vereinzelte zweireihige Zylinderepithelien zu sehen sind, in das einreihige niedrige Uterusepithel übergeht; zugleich treten typische Uterusdrüsen auf, so dass die Cervix histologisch hier gar nicht differenziert ist. 


\section{Uterushorn.}

Die Veränderungen der Uterusschleimhaut an der Inplantationsstelle des Eies während und nach der Gravidität sind schon wiederholt Gegenstand eingehender Arbeiten gewesen, so dass ihre Berücksichtigung hier überflüssig erscheint. Hingegen dürften hier einige Bemerkungen über die Uterusdrüsen während der Gravidität bei der Ratte am Platze sein, um sie gelegentlich mit. Veränderungen während der reinen Brunstform vergleichen $\mathrm{zu}$ können.

Während der Gravidität sind die Uterusdrüsen einerseits auf den Raum zwischen den Fruchtkammern, andererseits auf jenen Teil der Schleimhaut beschränkt, welcher der Dezidua reflexa gegenüberliegt und mit ihr die schmale Uterusspalte begrenzt. Letztere Schilderung wird verständlich, wenn wir daran erinnern, dass die Bildung einer Dezidua vera bei der Ratte ausbleibt und daher auch über den Fruchtsäcken ein schmaler Schleimhautstreifen unverändert erhalten wird. Die Drüsen, die an den beschriebenen Stellen zu sehen sind, scheinen einander näher gerückt, sind etwas gewunden und sezernieren in so reichem Masse Schleim, dass sowohl der Interstitialraum als auch die Uterusspalte stets mit Sekret erfüllt sind. Da bei der Ratte die Uterusspalte nie vollständig verklebt, so steht der Entleerung eines Teiles dieses Sekretes in die Cervix kein Hindernis entgegen.

Nach dem Wurfe sind die Drüsenlumina sehr weit, fast sekretleer und mit Leukocyten erfüllt.

Bei der Brunstratte waren die Drüsen etwas geschlängelt und die Sekretbildung eine sehr reichliche.

Tuben.

Dieses Organ bot nur beim Meerschweinchen eine Besonderheit, insofern kurz nach dem Wurf sehr zahlreiche Flimmerzellen die Gestalt von Becherzellen angenommen haben."

\section{Lenkocytose.}

Ratte. Während der vorangegangenen Beschreibung ist die sebr reichliche Auswanderung meist mehrkerniger weisser. Blutkörperchen als Begleiterscheinung der Zellveränderungen bereits wiederholt erwähnt worden. Es erübrigt nur noch hinzuzufügen, dass auch die Schleimhaut der Uterushörner nach dem Wurfe von zahlreichen Leukocyten durchsetzt ist, die an die Oberfläche wandern 
und ins Lumen austreten. Daselbst sind sie bis zum Ende der ersten Woche zu finden.

Demnach ist die Leukocytenauswanderung zur Zeit des Zellwechsels immer über den ganzen Genitalkanal bis zur Einmündung der Tuben, in denen sie nie beobachtet wird, ausgedehnt.

In der Vagina dürften sie nie ganz verschwinden, doch scheint ihre Anzahl zu Beginn der zweiten Woche vermindert zu sein, während sie vor und nach dem Wurfe am grössten ist.

In der Cervix harren sie in grösserer Anzahl, etwa so lange, wie im Uterushorn aus, verschwinden dann fast vollkommen und erscheinen mit Beginn der höhergradigen Verschleimung wieder.

Die vermehrte Auswanderung der Leukocyten trifft demnach stets mit dem Zellwechsel zusammen, und es erscheint daher die Annahme wahrscheinlich, dass sie beim Umbau der Schleimhaut eine Aufgabe zu erfülien haben.

Ihr Verhalten zu den Spermatozoen, die gerade zu einer Zeit, in der sie in grosser Menge auftreten, in den Genitalkanal gelangen, wird an anderer Stelle besprochen werden. Hier möge nur noch ibr Zusammentreffen mit dem $\mathrm{Ei}$ im Uterushorn kurz erwähnt werden. Nach den Untersuchungen Sobottas ${ }^{1}$ ) an der Maus findet die Inplantation des Eies erst am 5.-6. Tag statt, so dass das Ei relativ lange auf sein eigenes oder auf das Nährmaterial der umgebenden Flüssigkeit angewiesen ist. Es liegt demnach vielleicht nicht ausserhalb des Bereiches der Möglichkeit, die Leukocyten in einem $\mathrm{Zu}$ sammenhang mit der Erhaltung der Eies im Uterus bis zum Augenblick seiner Inplantation zu bringen.

Beim Kaninchen fehlt in meinen Präparaten stets die Leukocytenwanderung.

\section{Inhalt des Genitalkanals.}

Die Blutung während der Geburt ist infolge der Stielung ${ }^{2}$ ) der Plazenta eine sehr geringe und schon kurze Zeit nach dem Wurf auch mikroskopiseh nicht mehr nachzuweisen. Hingegen kommen Blutungen während der Gravidität häufig vor, und in zahlreichen hier untersuchten Tieren war der Cervikalkanal oder ein Stück des Uterus-

1) Die Befruchtung und Furchung des Eies bei der Maus. Arch. f. Anat. u. Entw. Bd. 45.

2) H. Strahl, Vom Uterus post partum. Ergeb. d. Anat. u. Entw. Bd. 15. 
Die Veränderungen der Genitalschleimhaut während der Gravidität etc. 569

hornes durch Blutfüllung stark ausgedehnt, ohne dass hierbei die Embryonen geschädigt worden wären.

Die Besprechung über den Schleim und das Sekret im Genitalkanal kann um so kürzer sein, als wir hierbei wiederholt an bereits Beschriebenes anknüpfen müssen.

Im engen Zusammenhang mit den Veränderungen der Schleimhaut wird die Vagina zur Zeit der Brunst, gleichgültig ob eine Gravidität vorausgegangen ist oder nicht, von einem Gebilde erfüllt, das. zum Teil den abgefallenen verschleimten Wandzellen seine Entstehung verdankt und zuerst von Héron-Royer ${ }^{1}$ ) und Lataste ${ }^{2}$, bei Pachyuromys Duprasi gefunden und von letzterem als „bouchon vaginal" bezeichnet wurde. Eine weitere Beschreibung dieses Pfropfes rührt von $\mathrm{Tafani}^{3}$ ) und später von Sobotta ${ }^{4}$ ) her, die beide die Maus untersuchten.

In Zentrum dieses Ballens liegt bei stattgehabter Kohabitation das erstarrte Sekret der männlichen Geschlechtsdrüsen, während die verschleimten Zellen die periphere Hülle bilden. Der Pfropf füllt in der Regel bloss die Vagina aus, doch erwähnt Sobotta einen Fall, bei welchem auch die beiden Uterushörner einer Maus bis zu den Tubenmündungen von demselben ausgedehnt waren. Nach den Angaben desselben Autors erweicht der anfangs sehr harte Pfropf sehr bald im Zentrum und ist in 20-30 Stunden ausgestossen.

Nach meinen Beobachtungen, die im Interesse einer Spermatozoenuntersuchung systematisch in verschiedenen Zeiten der Trächtigkeit ausgeführt wurden, kann ich der gegebenen Schilderung noch Folgendes hinzufügen. Bei einer Ratte, die 4 Tage nach dem Wurf obduziert wurde, war die Vagina noch stark von einem Pfropf ausgedehnt, der einen Ballen bereits veränderter Spermatozoen enthielt, und bei einer Ratte am 9. Tage nach der Konzeption war der Rest der in der Vagina liegenden Schleimmasse noch immer ziemlich ansehnlich. Während in der Mitte der Tragzeit die Schleim-

1) Zool. Anz. 28. November 1881 .

2) Sur le bouchon vaginal du Pachyuromys Duprasi. Zool. Anz. Bd. 5. 1882. Compt. rend. de la société de biol. 1888.

3) A. Tafani, Le fecondatione e la segmentatione studiate nelle uova dei topi. Acad. med. fisci Fiorent. 1888.

4) Die Befruchtung und Furchung des Eies bei der Maus. Arch. f. Anat. Bd. 45 . 
massen sehr gering sind, nehmen sie gegen das Ende der Gravidität wieder an Menge zu.

Diese Ergebnisse scheinen für gewisse Zeitbestimmungen bei der Untersuchung des Eies in der Tube und im Uterus nicht unwesentlich zu sein. Denn da der Augenblick des Koitus bei diesen Tieren fast nie beobachtet werden kann, sind viele Berechnungen mit Zuhilfenahme des Vaginapfropfes in der Voraussetzung, dass derselbe in sehr kurzer Zeit ausgestossen wird, ausgeführt worden. Die Beobachtung, dass derselbe 4 Tage und auch länger ziemlich unverändert bei der Ratte in der Vagina verweilen kann, sind vielleicht geeignet, einige Differenzen verschiedener Autoren über die Zeitpunkte der Eifurchungen usw. aufzuklären.

Die mikroskopische Untersuchung dieses Pfropfes zeigt, vom männlichen Geschlechtssekret abgesehen, neben Schleim, Detritus und zahlreichen Leukocyten, den jeweiligen Schleimhautveränderungen entsprechend, verschleimte Zellen oder Plattenepithelien in grösserer Menge. Ausserdem erkennt man neben dem typisch gefärbten Schleim noch eine eosinophyle leichtkörnige Sekretmasse, die in Streifen oder Ballen geformt ist und bald innig vermischt mit dem Schleim gefunden wird, bald scharf von demselben abgegrenzt erscheint. Dieses Sekret wird von den Drüsen des Uterus und den Schleimhautzellen der Cervix geliefert, und während dasselbe in den ersten Tagen nach dem Wurfe nur spärlich vertreten ist, bildet es vom Ende der zweiten Woche an den Hauptbestandteil des Vaginalinhaltes, um am Ende der Gravidität wieder von verschleimten Zellen verdrängt zu werden.

Im Lumen der Cervix liegen in den ersten Tagen nach dem Wurfe die abgestossenen Zellen neben Sekrettropfen und typischem Schleime. Später ist der Kanal mit etwas Sekret erfüllt.

Beim Kaninchen fehlt die Verschleimung und Desquamation der Wandzellen und dementsprechend auch der Schleimpfropf und die Rundzellenauswanderung. Fine geringe Menge einer zäben, bräunlich gefärbten Flüssigkeit sammelt sich in den Scheidengewölben an und besteht aus eosinophylen Sekrettropfen und Spermatozoen. Manchmal liegt ein etwas über Haselnuss grosser, ziemlich weicher Klumpen von grauer Farbe vor den Portiozapfen, der das geronnene Sekret der männlichen Geschlechtsdrüsen darstellt. 